\title{
¿La ciudadanía es responsable del manejo de la pandemia? Un análisis desde los determinantes sociales
}

\section{Are the citizens responsible for the pandemic management? An analysis from the social determinants}

\author{
Alvaro-Javier Idrovo ${ }^{1}$
}

Forma de citar: Idrovo AJ. ¿La ciudadanía responsable de la pandemia? Un análisis desde los determinantes sociales. Salud UIS. 2020; 52(4): 362-364. doi: http://dx.doi.org/10.18273/revsal.v52n4-2020001 (c) (1)

A finales de septiembre de 2020, cuando se escribe este editorial, Colombia como país ha superado el primer pico de la pandemia, y Santander está llegando al primer pico estimado en el modelo del Instituto Nacional de Salud. Han pasado varios eventos relacionados, directa o indirectamente, con la pandemia que se pueden resumir en un periodo de confinamiento temprano, que inició desde días después del diagnóstico del primer caso de SARSCoV-2 en una colombiana; luego empezó el desconfinamiento, desde el 1 de junio ${ }^{1}$, que se amplió con un segundo grupo de actividades económicas el 1 de septiembre, y que incluyó hechos importantes como los días sin IVA que buscaron reactivar la economía, y fueron noticia por las aglomeraciones que se presentaron en algunas ciudades. Si bien lo adecuado del manejo nacional es y será motivo de discusión ${ }^{1,2}$, las evidencias ya permiten señalar que no fue homogéneo. Departamentos como Antioquia, Tolima, Bogotá, Nariño y Valle del Cauca, parece tuvieron un desempeño de vigilancia en salud pública sobresaliente, mientras que otros departamentos incluyendo Santander ocuparon los últimos lugares, cuando se usa como indicador la calidad de los datos ${ }^{3}$.

Algo que ha sido notorio en el discurso gubernamental durante la pandemia, incluso desde los primeros momentos, es que la prevención depende de cada persona. Se indica que el uso de tapabocas, el lavado de manos frecuente y adecuado, y el distanciamiento físico resultan ser las acciones principales para manejar la pandemia. ¡Toda la responsabilidad en manos de cada persona! Esto que parece obvio ha sido secundado por personal de salud que repite el mismo discurso, quizá por una medicalización excesiva apoyada por los hallazgos epidemiológicos individualizadores, que si no son bien leídos ocultan los efectos de los determinantes sociales. Sin embargo, y sin quitarle la efectividad que sin duda tienen estas acciones en el manejo de la pandemia, es importante detenerse y analizar si realmente la ciudadanía es la única responsable del manejo de la pandemia. Un análisis más profundo, que parta desde el entendimiento de los determinantes sociales, fácilmente pondrá un valor relativo a esta afirmación. Así pues, veamos algunas evidencias que muestran claramente cómo ese discurso resulta ser culpabilizador ${ }^{4} \mathrm{e}$ injusto con la ciudadanía:

1. El confinamiento que se inició en Bogotá y luego se extendió por el país, ha sido la decisión política que más ha frenado la transmisión de SARS-CoV-2 en Colombia. La ocurrencia de casos y fallecidos antes del 1 de junio son evidencia suficiente para indicar que fue una medida útil, aunque heterogénea en su aplicación ${ }^{3}$; sin embargo, su gran limitación es que era insostenible a largo plazo. Si bien es una acción conocida desde siglos atrás, en sus expresiones de cuarentena de individuos sanos, o aislamiento de individuos infectados, lo que no resulta claro es si quienes tomaron la decisión entendieron que el objetivo principal era prepararse para cuando ocurriese el desconfinamiento. Las mejoras más importantes debían verse en vigilancia en salud pública,

1. Universidad Industrial de Santander. Bucaramanga, Colombia

Correspondencia: Javier Idrovo. Dirección: Cra. 32 29-31, Bucaramanga. Teléfono: +57 634 4000. Correo electrónico: idrovoaj@uis.edu.co 
incluyendo los diagnósticos rápidos en el laboratorio, y el alistamiento hospitalario, junto a un empoderamiento de la ciudadanía, mediante información, comunicación y educación.

2. No es lo mismo indicar en Colombia y en países más equitativos y con economías más sólidas, que la responsabilidad de la prevención es un asunto principalmente individual; eso implicaría un desconocimiento del contexto. ¿Qué proporción de la población colombiana tiene acceso a tapabocas, puede lavarse las manos frecuentemente, y puede darse el "lujo" de distanciarse físicamente? Para contestar ello es importante conocer que un alto porcentaje de la población colombiana no tiene acceso a agua potable $e^{5,6}$, tiene limitada conexión a internet $^{7} \mathrm{y}$ hace parte de los trabajadores informales, de los que viven diariamente del rebusque ${ }^{8}$. Es claro que una política pública, incluyendo si es sanitaria, no solo requiere el decretarla sino el de generar los contextos para que se pueda cumplir, y es precisamente allí donde los determinantes sociales han limitado su implementación, especialmente entre las poblaciones más marginadas de la sociedad.

3. Los efectos "colaterales" de la pandemia, que la llevan a configurar su dimensión mayor como sindemia9, están íntimamente relacionados con los determinantes sociales, que siempre han estado marcando las condiciones y necesidades de salud de los colombianos ${ }^{10,11}$. Las expresiones son muy variadas, pero sin duda las más alarmantes tienen que ver con las diversas formas de violencia, que incluyen el asesinato de líderes sociales ${ }^{12}$, las masacres y los asesinatos que han ocurrido en medio de protestas sociales ${ }^{13}$.

4. Uno de los fundamentos de la psicología social claramente indica que los comportamientos individuales están condicionados por el contexto social en el cual se encuentra el individuo ${ }^{14}$, por lo que el libre albedrío resulta más excepción que regla. Y si se está en un contexto precario, obviamente los comportamientos van a ser acordes a esas condiciones de vulnerabilidad.

5. El caso de Suecia, como país que no tuvo medidas de confinamiento y basó su estrategia en el distanciamiento físico bajo responsabilidad individual ${ }^{15}$, sigue siendo mal informado ${ }^{16}$ y seguido por muchos países, así no se haga de manera explícita. Sin embargo, nuevamente las diferencias demográficas, disponibilidad hospitalaria, servicios públicos, formas de trabajo, convivencia de adultos mayores con menores de edad, entre otros, en Suecia, hacen que una estrategia de este tipo no sea aplicable fácilmente en contextos latinoamericanos.

Como puede verse, el discurso gubernamental que descarga la responsabilidad del manejo de la pandemia en las personas, resulta incoherente, injusto y abre la posibilidad de justificaciones del mal manejo gubernamental de la pandemia por acción y, sobre todo, por omisión. Es imperioso que se tenga claro esta situación, porque los gobernantes están precisamente en esos cargos para buscar el bien de la sociedad, y es claro que entre los bienes, tanto la vida como la salud son prioritarios. La economía ya sabemos que se puede recuperar, con una fuerte inversión de dineros públicos ${ }^{16}$.

\section{Referencias}

1. De la Hoz-Restrepo F, Alvis-Zakzuk NJ, De la Hoz-Gómez JF, De la Hoz-Gómez A, Gómez del Corral L, AlvisGuzmán N. Is Colombia an example of successful containment of the COVID-19 2020 pandemic? A critical analysis of the epidemiological data. 2020. Int J Infect Dis. 2020; 99: 522-529. doi: 10.1016/j.ijid.2020.08.017

2. Manrique-Hernández EF, Moreno-Montoya J, Hurtado-Ortíz A, Prieto-Alvarado FE, Idrovo AJ. Desempeño del sistema de vigilancia colombiano durante la pandemia de COVID-19: evaluación rápida de los primeros 50 días. Biomédica 2020; 40(supl.2). doi: https://doi.org/10.7705/biomedica.5582

3. Hurtado-Ortiz A, Moreno-Montoya J, Prieto-Alvarado FE, Idrovo AJ. Evaluación comparativa de la vigilancia en salud pública de COVID-19 en Colombia: primer semestre. Biomédica 2020; 40(supl.2). doi: https://doi. org/10.7705/biomedica.5812

4. Krieger N. Got theory? On the 21st c. Ce rise of explicit use of epidemiologic theories of disease distribution: a review and ecosocial analysis. Curr Epidemiol Rep 2014; 1: 45-56. doi: 10.1007/s40471-013-0001-1

5. Guzmán BL, Nava G, Díaz Bevilacqua P. La calidad del agua para consumo humano y su asociación con la morbimortalidad en Colombia, 2008-2012. Biomédica 2015; 35(Sup2): 177-190. https://doi.org/10.7705/ biomedica.v35i0.2511

6. Guzmán BL, Nava G, Bavilacqua PD. Vigilancia de la calidad del agua para consumo humano en Colombia: desafíos para la salud ambiental. Rev Fac Nac Salud Pública 2016; 34(2): 175-183. doi: https://dx.doi. org/10.17533/udea.rfnsp.v34n2a06

7. Botello Peñaloza HA. Determinantes del acceso a Internet en Colombia. Ánfora. 2014; 21(37): 21-36. 
8. Cárdenas J, Robles-Rivera F, Martínez-Vallejo D. Élites empresariales y desigualdad en tiempos de pandemia en América Latina. Rev Esp Sociol. 2020; 29(3): http://dx.doi.org/10.2139/ssrn.3620577

9. Horton R. COVID-19 is not a pandemic. Lancet 2020; 396: 874. doi: https://doi.org/10.1016/S01406736(20)32000-6

10. Idrovo AJ, Ruíz-Rodríguez M. Una visión global a la salud de la población colombiana: rol de los macrodeterminantes sociales. Biomédica 2007; 27(3): 333-344. doi: https://doi.org/10.7705/biomedica.v27i3.196

11. Observatorio Nacional de Salud. Desigualdades sociales en salud en Colombia. Bogotá DC: Instituto Nacional de Salud; 2015.

12. Idrovo AJ. Comment on "I can't breathe - race, violence and COVID-19". Ann Surg 2020 [online ahead of print]. doi: 10.1097/SLA.0000000000004448

13. Idrovo AJ, Fernández-Niño JA. Hacia una epidemiología de las protestas sociales: principales lesiones y efectos de los gases lacrimógenos. Rev Univ Ind Santander. Salud 2020; 52(1): 5-6. doi: https://doi.org/10.18273/revsal. v52n1-2020001

14. Rodrigues A, Leal Assmar EM, Jablonski B. Psicologia social 29a ed. Petrópolis: Editora Vozes; 2000.

15. Kamerlin SCL, Kasson PM. Managing coronavirus disease 2019 spread with voluntary public health measures: Sweden as a case study for pandemic control. Clin Infect Dis 2020:ciaa864. doi: https://doi.org/10.1093/cid/ ciaa864

16. Irwin RE. Misinformation and de-contextualization: international media reporting on Sweden and COVID-19. Global Health 2020; 16: 62 doi: https://doi.org/10.1186/s12992-020-00588-x

17. Baldwin R, Weder di Mauro B. Economics in the time of COVID-19. London: Centre for Economic Policy Research; 2020. 